Bull. Austral. Math. Soc.

Vol. 45 (1992) [241-248]

\title{
VOLUME COMPARISON OF BISHOP-GROMOV TYPE
}

\author{
SUNGYUN LeE
}

\begin{abstract}
Bishop-Gromov type comparison theorems for the volume of a tube about a submanifold of a complete Riemannian manifold whose Ricci curvature is bounded from below are proved. The Kähler analogue is also proved.
\end{abstract}

\section{INTRODUCTION}

In Riemannian geometry, it is a fundamental question to ask how the geometric invariants of Riemannian manifolds are influenced by curvature restrictions. The volume of a geodesic ball is one of the basic invariants, for which the Bishop-Gromov comparison theorem is well-known (see $[1,6])$.

In this article we prove Bishop-Gromov type comparison theorems for the volume of a tube about a submanifold of a complete Riemannian manifold whose Ricci curvature is bounded from below. The Kähler analogue is also proved.

To be more specific let $M$ be a complete Riemannian manifold of dimension $n$ and let $P \subset M$ be a topologically embedded connected submanifold of dimension $q$ with compact closure. For $r \geqslant 0$ let $V_{P}^{M}(r)$ denote the $n$-dimensional volume of a tube of radius $r$ about $P$ and let $A_{P}^{M}(r)$ denote the $(n-1)$-dimensional volume of its boundary. Let $\lambda$ be a constant which may be positive, negative, or zero and let $K^{n}(\lambda)$ denote the $n$-dimensional space of constant curvature $\lambda$.

THEOREM 1. Let $P \subset M$ and suppose that the Ricci curvature $\rho^{M}$ of $M$ satisfies $\rho^{M} \geqslant(n-1) \lambda=\rho^{K^{n}(\lambda)}$. Let $\bar{P}$ denote a $q$-dimensional totally geodesic submanifold of $K^{n}(\lambda)$ such that volume $(P)=$ volume $(P)$.

(i) If $q=\operatorname{dim} P=0$, then $V_{m}^{M}(t) \leqslant V_{\bar{m}}^{K^{n}(\lambda)}(t)$ and $V_{m}^{M}(t) / V_{\bar{m}}^{K^{n}(\lambda)}(t)$ is a nonincreasing function of $t$ for $0<t \leqslant e_{c}(m)$. Here $P$ is a point $m \in M$ and $e_{c}(m)$ is the minimal distance to the cut locus of $m$.

(ii) If $1 \leqslant q \leqslant n-2$, then for any $\varepsilon>0$ there is small $t_{0}>0$ depending on $\varepsilon$ and $P \subset M$ such that $V_{P}^{M}\left(t, t_{0}\right) \leqslant(1+\varepsilon) C\left(t_{0}\right) V_{\bar{m}}^{K^{n}(\lambda)}\left(t, t_{0}\right)$ for $t_{0} \leqslant t \leqslant e_{c}(P)$, where $V_{P}^{M}\left(t, t_{0}\right)=V_{P}^{M}(t)-V_{P}^{M}\left(t_{0}\right)$ and $C\left(t_{0}\right)=$

Received 21 March 1919

This research was partially supported by KOSEF

Copyright Clearance Centre, Inc. Serial-fee code: 0004-8729/92 \$A2.00+0.00. 
$A_{\bar{P}}^{K^{n}(\lambda)}\left(t_{0}\right) / A_{\bar{m}}^{K^{n}(\lambda)}\left(t_{0}\right)$. Moreover $V_{P}^{M}\left(t, t_{0}\right) / V_{\bar{m}}^{K^{n}(\lambda)}\left(t, t_{0}\right)$ is a nonincreasing function of $t$ for $t_{0} \leqslant t \leqslant e_{c}(P)$. Here $e_{c}(P)$ is the minimal focal distance of $P$ in $M$.

(iii) If $q=n-1$ and $P$ is minimal, then $V_{P}^{M}(t) \leqslant V_{\bar{P}}^{K^{n}(\lambda)}(t)$ and $V_{P}^{M}(t) / V_{\bar{P}}^{K^{n}(\lambda)}(t)$ is a nonincreasing function of $t$ for $0 \leqslant t \leqslant e_{c}(P)$.

REMARK. Observe that the right-hand sides of inequalities in (i), (ii), (iii) do not depend on the embedding of $P$ into $M$. (i) is just the Bishop-Gromov comparison theorem for geodesic balls. If $\operatorname{dim} P \geqslant 1$, then for sufficiently small $r>0, V_{P}^{M}(r)$ depends strongly on $P$ as the power series expansion in [5] shows. This fact forces us to consider $V_{P}^{M}\left(t, t_{0}\right)$ in (ii). Even though $t_{0}>0$ depends on $\varepsilon$ and $P \subset M$ in (ii), sufficiently small $t_{0}$ always satisfies the inequality. Also observe that $t \mapsto$ $V_{P}^{M}(t) / V_{\bar{m}}^{K^{n}(\lambda)}(t)$ is nonincreasing for $0<t \leqslant e_{c}(P)$ in (ii). When $P$ is a hypersurface of $M$, an additional assumption on $P$ (that is, $P$ is minimal) is needed to get the global volume comparison in (iii), since the principal curvatures of $P$ strongly affects $V_{P}^{M}(r)$ for relatively large $r$.

Next, to state the Kähler analogue of Theorem 1, let $M$ be a complete Kähler manifold of real dimension $2 n$. Let $K_{h}^{M}\left(K_{a h}^{M}\right)$ denote the holomorphic (antiholomorphic) sectional curvature of $M$. The antiholomorphic Ricci curvature $\rho_{a h}^{M}$ of $M$ is the sum of antiholomorphic sectional curvatures (see for example [2]). Let $P \subset M$ be a topologically embedded connected complex submanifold of real dimension $2 q$ with compact closure.

Theorem 2. Let $P \subset M$ and suppose that $K_{h}^{M} \geqslant 4 \lambda$ and $\rho_{a h}^{M} \geqslant(2 n-2) \lambda$. Let $\vec{P}$ denote a totally geodesic complex submanifold of real dimension $2 q$ of $K_{h}^{n}(\lambda)$ such that voiume $(P)=\operatorname{volume}(P)$, where $K_{h}^{n}(\lambda)$ is a Kähler manifold of complex dimension $n$ with constant holomorphic sectional curvature $4 \lambda$.

(i) If $q=0$ (that is, $P$ is a point $m \in M$ ), then $V_{m}^{M}(t) \leqslant V_{\bar{m}}^{K_{h}^{n}(\lambda)}(t)$ and $V_{m}^{M}(t) / V_{\bar{m}}^{K_{h}^{n}(\lambda)}(t)$ is a nonincreasing function of $t$ for $0<t \leqslant e_{c}(m)$.

(ii) If $1 \leqslant q \leqslant n-2$, then for any $\varepsilon>0$ there is small $t_{0}>0$ depending on $\varepsilon$ and $P \subset M$ such that $V_{P}^{M}\left(t, t_{0}\right) \leqslant(1+\varepsilon) C\left(t_{0}\right) V_{\bar{m}}^{K_{h}^{n}(\lambda)}\left(t, t_{0}\right)$ for $t_{0} \leqslant t \leqslant e_{c}(P)$, where $C\left(t_{0}\right)=A_{\bar{P}}^{K_{h}^{n}(\lambda)}\left(t_{0}\right) / A_{\bar{m}}^{K_{h}^{n}(\lambda)}\left(t_{0}\right)$. Moreover $V_{P}^{M}\left(t, t_{0}\right) / V_{\bar{m}}^{K_{h}^{n}(\lambda)}\left(t, t_{0}\right)$ is a nonincreasing function of $t$ for $t_{0} \leqslant t \leqslant$ $e_{c}(P)$.

(iii) If $q=n-1$ (that is, $P$ is a complex hypersurface of $M$ ), then $V_{P}^{M}(t) \leqslant$ $V_{\bar{P}}^{K_{h}^{n}(\lambda)}(t)$ and $V_{P}^{M}(t) / V_{\bar{P}}^{K_{h}^{n}(\lambda)}(t)$ is a nonincreasing function of $t$ for $0 \leqslant$ $t \leqslant e_{c}(P)$. 
REMARK. (i) is the result essentially due to Nayatani [7] (see also [4, page 194]). In (ii) $V_{P}^{M}(t) / V_{\bar{m}}^{K_{h}^{n}(\lambda)}(t)$ is also a nonincreasing function of $t$ for $0<t \leqslant e_{c}(P)$.

We shall prove these theorems following the ideas in [4]. In Section 2 we review some preliminary results. The proofs of theorems are given in Section 3.

\section{Preliminaries $[3,4]$}

Let $M$ be a complete Riemannian manifold of dimension $n$ and let $P \subset M$ be a topologically embedded submanifold of dimension $q$ which is relatively compact.

Let $t \mapsto \gamma(t)$ be a unit speed geodesic in $M$ normal to $P$ with $\gamma(0)=p \in P$. Assume that $t \geqslant 0$ is less than or equal to the distance between $P$ and its nearest focal point. Denote by $S(t)$ the second fundamental form at the point $\gamma(t)$ of the tubular hypersurface at a distance $t$ from $P$. Also let $R(t): M_{\gamma(t)} \rightarrow M_{\gamma(t)}$ be the symmetric linear transformation defined by $\langle R(t) x, y\rangle=R_{\gamma^{\prime}(t) x \gamma^{\prime}(t) y}$, where $\langle$,$\rangle and R^{M}$ are the metric and the Riemannian curvature tensor field of $M$ respectively, $M_{\gamma(t)}$ denotes the tangent space to $M$ at $\gamma(t)$, and $x, y \in M_{\gamma(t)}$. Then $S(t)$ satisfies the differential equation

$$
S^{\prime}(t)=S(t)^{2}+R(t)
$$

Let $\omega$ be the Riemannian volume form of $M$, and let $\left(x_{1}, \ldots, x_{n}\right)$ be a system of Fermi coordinates such that

$$
\omega\left(\frac{\partial}{\partial x_{1}} \wedge \cdots \wedge \frac{\partial}{\partial x_{n}}\right)(\gamma(t))>0
$$

For $u \in P_{p}^{\perp}$ with $\|u\|=1$, put

$$
\theta_{u}(t)=\omega\left(\frac{\partial}{\partial x_{1}} \wedge \cdots \wedge \frac{\partial}{\partial x_{n}}\right)(\gamma(t))
$$

Then $\theta_{u}(0)=1$ and

$$
\frac{\theta_{u}^{\prime}(t)}{\theta_{u}(t)}=-\left(\frac{n-q-1}{t}+\operatorname{tr} S(t)\right)
$$

Let $V_{P}^{M}(r)=n$-dimensional volume of $\{m \in M \mid d(m, P) \leqslant r\}$ and $A_{P}^{M}(r)=(n-1)$ dimensional volume of $\{m \in M \mid d(m, P)=r\}$. Then

$$
A_{P}^{M}(t)=\int_{P} \int_{S^{n-q-1}(1)} t^{n-q-1} \theta_{u}(t) d u d P
$$

and

$$
V_{P}^{M}(r)=\int_{0}^{r} A_{P}^{M}(t) d t
$$


where $S^{n-q-1}(1)$ is the unit sphere in $P_{p}^{\perp}$. If $\bar{P} \subset K^{n}(\lambda)$ is a totally geodesic submanifold,

$$
A_{\bar{P}}^{K^{n}(\lambda)}(t)=\frac{2 \pi^{(n-q) / 2}}{\Gamma\left(\frac{n-q}{2}\right)}\left(\frac{\sin t \sqrt{\lambda}}{\sqrt{\lambda}}\right)^{n-q-1}(\cos t \sqrt{\lambda})^{q} \text { volume }(P),
$$

and if $\bar{P} \subset K_{h}^{n}(\lambda)$ is a totally geodesic complex submanifold,

$$
A_{\bar{P}}^{K_{h}^{n}(\lambda)}(t)=\frac{2 \pi^{n-q}}{(n-q-1) !}\left(\frac{\sin t \sqrt{\lambda}}{\sqrt{\lambda}}\right)^{2 n-2 q-1}(\cos t \sqrt{\lambda})^{2 q+1} \text { volume }(P) .
$$

In (5) and (6) we interpret $(\sin t \sqrt{\lambda}) / \sqrt{\lambda}$ as $(\sinh t \sqrt{-\lambda}) / \sqrt{-\lambda}$ (respectively $t$ ) when $\lambda<0$ (respectively $\lambda=0$ ).

\section{Proof of ThEOREMS}

We will use many results of [4]. The proof of Theorem 2 is similar to but a little more complicated than that of Theorem 1.

Proof of Theorem 1: (i) This is just the Bishop-Gromov volume comparison theorem for geodesic balls (see $[1,4,6]$ ). Observe that the proof of case (ii) gives us (i) easily.

(ii) Let $1 \leqslant q=\operatorname{dim} P \leqslant n-2$, and $0<t \leqslant e_{c}(P)$.

Then $t \rightarrow \theta_{u}(t) /(\sin t \sqrt{\lambda} / t \sqrt{\lambda})^{n-1} t^{q}$ is nonincreasing (see [4, p. 181]). For any $\varepsilon>0$, there is small $t_{0}>0$ depending on $\varepsilon$ and $P \subset M$ such that $\theta_{u}\left(t_{0}\right) \leqslant(1+\varepsilon)\left(\sin t_{0} \sqrt{\lambda} / t_{0} \sqrt{\lambda}\right)^{n-q-1}\left(\cos t_{0} \sqrt{\lambda}\right)^{q}$ since $\lim _{t_{0} \rightarrow 0} \theta_{u}\left(t_{0}\right)=1$.

Then we have for $0<t_{0} \leqslant s \leqslant t$

$$
s^{n-q-1} \theta_{u}(s) \leqslant(1+\varepsilon) t_{0}^{n-q-1}\left(\frac{\sin t_{0} \sqrt{\lambda}}{t_{0} \sqrt{\lambda}}\right)^{n-q-1}\left(\cos t_{0} \sqrt{\lambda}\right)^{q} /\left(\frac{\sin t_{0} \sqrt{\lambda}}{\sqrt{\lambda}}\right)^{n-1}
$$

and

$$
t^{n-q-1} \theta_{u}(t)\left(\frac{\sin s \sqrt{\lambda}}{\sqrt{\lambda}}\right)^{n-1} \leqslant s^{n-q-1} \theta_{u}(s)\left(\frac{\sin t \sqrt{\lambda}}{\sqrt{\lambda}}\right)^{n-1} .
$$

Integrating (7) over the unit sphere $S^{n-q-1}(1)$ in $P_{p}^{\perp}$ and using (3), (5), we obtain

$$
A_{P}^{M}(s) \leqslant(1+\varepsilon) \frac{A_{\bar{P}}^{K^{n}(\lambda)}\left(t_{0}\right)}{A_{\bar{m}}^{K^{n}(\lambda)}\left(t_{0}\right)} A_{\bar{m}}^{K^{n}(\lambda)}(s)
$$


Then integrating (9) with respect to $s$ from $t_{0}$ to $t$ and using (4) we get

$$
V_{P}^{M}\left(t, t_{0}\right) \leqslant(1+\varepsilon) C\left(t_{0}\right) V_{\bar{m}}^{K^{n}(\lambda)}\left(t, t_{0}\right),
$$

where $C\left(t_{0}\right)=A_{\bar{P}}^{K^{n}(\lambda)}\left(t_{0}\right) / A_{\bar{m}}^{K^{n}(\lambda)}\left(t_{0}\right)$. Similarly from (8) we obtain

$$
V_{P}^{M}\left(t, t_{0}\right) A_{\bar{m}}^{K^{n}(\lambda)}(t) \geqslant V_{\bar{m}}^{K^{n}(\lambda)}\left(t, t_{0}\right) A_{P}^{M}(t) .
$$

But (11) implies that

$$
\begin{gathered}
\frac{d}{d t}\left\{\frac{V_{P}^{M}\left(t, t_{0}\right)}{V_{\bar{m}}^{K^{n}(\lambda)}\left(t, t_{0}\right)}\right\}=\frac{1}{\left\{V_{\bar{m}}^{K^{n}(\lambda)}\left(t, t_{0}\right)\right\}^{2}} \times \\
\left(V_{\bar{m}}^{K^{n}(\lambda)}\left(t, t_{0}\right) A_{P}^{M}(t)-V_{P}^{M}\left(t, t_{0}\right) A_{\bar{m}}^{K^{n}(\lambda)}(t)\right) \leqslant 0 .
\end{gathered}
$$

(iii) Let $P$ be a minimal hypersurface of $M$ and $0 \leqslant t \leqslant e_{c}(P)$. Then Lemma 8.28 in $[4$, page 181] shows that

$$
\theta_{u}(t) \leqslant(\cos t \sqrt{\lambda})^{n-1}
$$

and

$$
\theta_{u}(t)(\cos s \sqrt{\lambda})^{n-1} \leqslant \theta_{u}(s)(\cos t \sqrt{\lambda})^{n-1} .
$$

Integrating (12) and (13) we get

$$
V_{P}^{M}(t) \leqslant V_{\bar{P}}^{K^{n}(\lambda)}(t)
$$

and

$$
V_{P}^{M}(t) A_{\bar{P}}^{K^{n}(\lambda)}(t) \geqslant A_{P}^{M}(t) V_{\bar{P}}^{K_{n}(\lambda)}(t)
$$

But (15) implies that $d\left(V_{P}^{M}(t) / V_{\bar{P}}^{K^{n}(\lambda)}(t)\right) / d t \leqslant 0$. This completes the proof of Theorem 1 .

Proof of TheOREM 2: Let $\left\{e_{1}, e_{1^{*}}, \ldots, e_{n}, e_{n^{*}}\right\}$ be an orthonormal basis of the tangent space $M_{p}$ such that $e_{i^{*}}=J e_{i}$ and $e_{1}, e_{1^{*}}, \ldots, e_{q}, e_{q^{*}}$ are tangent to $P$. Let $\gamma(t)$ be a unit speed geodesic with $\gamma(0)=p$ and $\gamma^{\prime}(0)=u=e_{n}$. Extend $e_{1}, e_{1 *}, \ldots, e_{n}, e_{n} *$ to orthonormal vector fields $E_{1}(t), E_{1 *}(t), \ldots, E_{n}(t), E_{n^{*}}(t)$ along $\gamma$ so that $E_{n}(t)=$ $\gamma^{\prime}(t)$ and the other $E_{i}(t)$ diagonalise $S(t)$. Consider the functions

$$
f_{i}(t)=\left\langle S(t) E_{i}(t), E_{i}(t)\right\rangle, \quad i \neq n .
$$


Taking the derivative of both sides of (16) and using (1) and the Cauchy-Schwarz inequality, we get

$$
\begin{aligned}
f_{i}^{\prime}(t) & =\left\langle S^{\prime} E_{i}, E_{i}\right\rangle=\left\langle S^{2} E_{i}+R E_{i}, E_{i}\right\rangle=\left\|S E_{i}\right\|^{2}+\left\langle R E_{i}, E_{i}\right\rangle \\
& \geqslant\left\langle S E_{i}, E_{i}\right\rangle^{2}+\left\langle R E_{i}, E_{i}\right\rangle=f_{i}^{2}(t)+\left\langle R(t) E_{i}(t), E_{i}(t)\right\rangle .
\end{aligned}
$$

Let

$$
f(t)=\frac{1}{2 n-2} \sum_{i=1}^{(n-1)^{*}} f_{i}(t)
$$

Since $\rho_{a h}^{M}=(2 n-2) f(t) \geqslant(2 n-2) \lambda$ and $K_{b}^{M} \geqslant 4 \lambda$, we have

$$
f^{\prime}(t) \geqslant f^{2}(t)+\lambda
$$

and

$$
f_{n^{*}}^{\prime}(t) \geqslant f_{n^{*}}^{2}(t)+4 \lambda .
$$

The differential inequalities (19) and (20) can be solved explicitly (see for example [4, pp.174-175]).

(i) This is the result essentially due to Nayatani [7]. Observe that the proof of case (ii) gives us (i) easily.

(ii) Let $1 \leqslant q=\operatorname{dim} P / 2 \leqslant n-2$ and $0<t \leqslant e_{c}(P)$. Since $f(0)=f_{n^{*}}(0)=-\infty$, we have from (19) and (20)

$$
f(t) \geqslant-\sqrt{\lambda} \cot t \sqrt{\lambda} \text { and } f_{n^{*}}(t) \geqslant-2 \sqrt{\lambda} \cot 2 t \sqrt{\lambda} .
$$

Summing the functions $f_{i}(t)$, we find that

$$
\operatorname{tr} S(t) \geqslant-(2 n-2) \sqrt{\lambda} \cot t \sqrt{\lambda}-2 \sqrt{\lambda} \cot 2 t \sqrt{\lambda} .
$$

From (2) and (21) we obtain

$$
\frac{d}{d t} \ln \theta_{u}(t)=-\frac{2 n-2 q-1}{t}-t r S(t) \leqslant \frac{d}{d t} \ln \alpha(t),
$$

where

$$
\alpha(t)=\left(\frac{\sin t \sqrt{\lambda}}{t \sqrt{\lambda}}\right)^{2 n-2 q-1}(\sin t \sqrt{\lambda})^{2 q} \cos t \sqrt{\lambda} .
$$

Then $d\left(\ln \left(\theta_{u}(t) / \alpha(t)\right)\right) / d t \leqslant 0$, and $\theta_{u}(t) / \alpha(t)$ is a nonincreasing function of $t$. For any $\varepsilon>0$, there is $t_{0}>0$ depending on $\varepsilon$ and $P \subset M$ such that $\theta_{u}\left(t_{0}\right) \leqslant$ 
$(1+\varepsilon)\left(\sin \left(t_{0} \sqrt{\lambda}\right) /\left(t_{0} \sqrt{\lambda}\right)\right)^{2 n-2 q-1}\left(\cos t_{0} \sqrt{\lambda}\right)^{2 q+1}$ since $\lim _{t_{0} \rightarrow 0} \theta_{u}\left(t_{0}\right)=1$. Then we have for $0<t_{0} \leqslant s \leqslant t$

$$
s^{2 n-2 q-1} \theta_{u}(s) \leqslant(1+\varepsilon) \frac{A_{\bar{m}}^{K_{h}^{n}(\lambda)}(s)}{A_{\bar{m}}^{K_{h}^{n}(\lambda)}\left(t_{0}\right)} t_{0}^{2 n-2 q-1}\left(\frac{\sin t_{0} \sqrt{\lambda}}{t_{0} \sqrt{\lambda}}\right)^{2 n-2 q-1}\left(\cos t_{0} \sqrt{\lambda}\right)^{2 q+1}
$$

and

$$
\begin{aligned}
& t^{2 n-2 q-1} \theta_{u}(t)\left(\frac{\sin s \sqrt{\lambda}}{\sqrt{\lambda}}\right)^{2 n-2 q-1}(\cos s \sqrt{\lambda}) \\
\leqslant & s^{2 n-2 q-1} \theta_{u}(s)\left(\frac{\sin t \sqrt{\lambda}}{\sqrt{\lambda}}\right)^{2 n-2 q-1}(\cos t \sqrt{\lambda}) .
\end{aligned}
$$

Integrating (22) using (3) we obtain

$$
A_{P}^{M}(t) \leqslant(1+\varepsilon) \frac{A_{\bar{P}}^{K_{h}^{n}(\lambda)}\left(t_{0}\right)}{A_{\bar{m}}^{K_{h}^{n}(\lambda)}\left(t_{0}\right)} A_{\bar{m}}^{K_{h}^{n}(\lambda)}(t)
$$

Integrating (24) with respect to $s$ from $t_{0}$ to $t$ we get

$$
V_{P}^{M}\left(t, t_{0}\right) \leqslant(1+\varepsilon) C\left(t_{0}\right) V_{\bar{m}}^{K_{h}^{n}(\lambda)}\left(t, t_{0}\right),
$$

where $C\left(t_{0}\right)=A_{\bar{P}}^{K_{h}^{n}(\lambda)}\left(t_{0}\right) / A_{\bar{m}}^{K_{h}^{n}(\lambda)}\left(t_{0}\right)$. Similarly, from (23) we obtain

$$
V_{P}^{M}\left(t, t_{0}\right) A_{\frac{m}{m}}^{K_{h}^{n}(\lambda)}(t) \geqslant A_{P}^{M}(t) V_{\bar{m}}^{K_{h}^{n}(\lambda)}\left(t, t_{0}\right)
$$

But (26) implies that $d\left(V_{P}^{M}\left(t, t_{0}\right) / V_{\bar{m}}^{K_{h}^{n}(\lambda)}\left(t, t_{0}\right)\right) / d t \leqslant 0$.

(iii) Let $P$ be a complex hypersurface of $M$ and $0 \leqslant t \leqslant e_{c}(P)$. Then $f_{i}(0)$, $1 \leqslant i \leqslant(n-1)^{*}$, are finite and $f(0)=0$ since the mean curvature vector fields of a Kähler submanifold of $M$ vanishes. Hence we obtain from (19) and (20)

$$
f(t) \geqslant \sqrt{\lambda} \cot t \sqrt{\lambda} \text { and } f_{n^{*}}(t) \geqslant-2 \sqrt{\lambda} \cot 2 t \sqrt{\lambda} \text {. }
$$

Summing the functions $f_{i}(t)$, we find that

$$
\operatorname{tr} S(t) \geqslant 2(n-2) \sqrt{\lambda} \cot t \sqrt{\lambda}-2 \sqrt{\lambda} \cot 2 t \sqrt{\lambda} \text {. }
$$

From (2) and (27) it follows that

$$
\frac{d}{d t} \ln \theta_{u}(t)=-\frac{1}{t}-\operatorname{tr} S(t) \leqslant \frac{d}{d t} \ln \beta(t),
$$


where $\beta(t)=(\sin t \sqrt{\lambda} / t \sqrt{\lambda})(\cos t \sqrt{\lambda})^{2 n-1}$. Then $d \ln \left(\theta_{u}(t) / \beta(t)\right) / d t \leqslant 0$, and $\theta_{u}(t) / \beta(t)$ is a nonincreasing function of $t$, whose value for $t=0$ is 1 , whence

$$
\theta_{u}(t) \leqslant \beta(t)
$$

and

$$
t \theta_{u}(t) A_{\bar{P}}^{K_{h}^{n}(\lambda)}(s) \leqslant s \theta_{u}(s) A_{\bar{P}}^{K_{h}^{n}(\lambda)}(t)
$$

for $0 \leqslant s \leqslant t$, follows. Integrating (28) using (3), (4), we get

$$
V_{P}^{M}(t) \leqslant V_{\bar{P}}^{K_{h}^{n}(\lambda)}(t)
$$

Similarly integrating (29), we obtain

$$
V_{P}^{M}(t) A_{\bar{P}}^{K_{h}^{n}(\lambda)}(t) \geqslant A_{P}^{M}(t) V_{\bar{P}}^{K_{h}^{n}(\lambda)}(t) .
$$

But (31) implies that $V_{P}^{M}(t) / V_{\bar{P}}^{K_{h}^{n}(\lambda)}(t)$ is a nonincreasing function of $t$. This completes the proof of Theorem 2.

\section{REFERENCES}

[1] R.L. Bishop and R.J. Crittenden, Geometry of manifolds (Academic Press, New York, 1964).

[2] F. Giménez and V. Miquel, 'Volume estimates for real hypersurfaces of a Kaehler manifold with strictly positive holomorphic sectional and antiholomorphic Ricci curvatures', Pacific J. Math. 142 (1990), 23-39.

[3] A. Gray, 'Comparison theorems for the volumes of tubes as generalizations of the Weyl tube formula', Topology 21 (1982), 201-228.

[4] A. Gray, Tubes (Addison-Wesley, New York, 1990).

[5] A. Gray and L. Vanhecke, 'The volume of tubes in a Riemannian manifold', Rend. Sem. Math. Univ. Politec. Torino 39 (1983), 1-50.

[6] M. Gromov, Structures Métriques pour les Variétés riemanniennes (Cédic Nathan, Paris, 1981).

[7] S. Nayatani, 'On the volume of positively curved Kähler manifolds', Osaka J. Math. 25 (1988), 223-231.

Department of Mathematics and Mathematics Research Center

KAIST

Taejon, 305-701

Korea 